Supporting Information 


\section{Revisiting Metal-organic Frameworks for}

\section{Oxygen Evolution: A Case Study}

Younes Mousazade, ${ }^{a}$ Mohammad Reza Mohammadi, ${ }^{b}$ Petko Chernev, ${ }^{c, d}$ Robabeh Bagheri,e Zhenlun Song, ${ }^{f}$ Holger Dau, ${ }^{d}$ and Mohammad Mahdi Najafpour*a

aDepartment of Chemistry, Institute for Advanced Studies in Basic Sciences (IASBS), Zanjan, 45137-66731, Iran

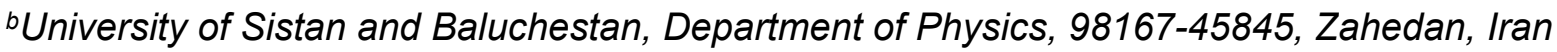

‘Uppsala University, Department of Chemistry - Angströmlaboratoriet, Lägerhyddsvägen 1, 75120 Uppsala, Sweden

${ }^{d}$ Freie Universität Berlin, Fachbereich Physik, Arnimallee 14, 14195 Berlin, Germany

eSchool of Physical Science and Technology, College of Energy, Soochow Institute for Energy and Materials Innovations and Key Laboratory of Advanced Carbon Materials and Wearable Energy Technologies of Jiangsu Province, Soochow University, Suzhou 215006, China

${ }^{f}$ Key Laboratory of Marine Materials and Related Technologies, Zhejiang Key Laboratory of Marine Materials and Protective Technologies, Ningbo Institute of Materials Technology and Engineering, Chinese Academy of Sciences, Ningbo 315201, China

Corresponding author: Tel: (+98) 243315 3201; E-mail: mmnajafpour@iasbs.ac.ir (MMN) 


\section{Table of contents}

Title

Page

Experimental Section

S4,S5

Figure S1 (FTIR spectra)

Figure S2 (SEM images of MOF 1)

Figure S3 (SEM images MOF 1 after one-hour amperometry)

S8

Figure S4 (HRTEM images of MOF 1)

Figure S5 (HRTEM images MOF 1 after one-hour amperometry)

Figure S7 k3-weighted EXAFS oscillations of the NiCo-UMOF1, MOF1 and MOF1 after S13 operation 


\section{Experimental Section}

\section{Materials}

All reagents and solvents were obtained from commercial sources and used without further purification, unless otherwise stated $\mathrm{KOH}$ pellets, $\mathrm{NiCl}_{2} \cdot 6 \mathrm{H}_{2} \mathrm{O}, \mathrm{CoCl}_{2} \cdot 6 \mathrm{H}_{2} \mathrm{O}$, triethylamine (TEA), dimethylformamide (DMF), and ethanol were purchased from Merck and fluorine-doped tin oxide coated glass (FTO), Nafion 117 solution (10\%), and were purchased from Sigma-Aldrich. Glassy carbon disk working electrodes were purchased from $\mathrm{CH}$ Instruments. For the experiments, milli- $\mathrm{Q}$ water $\left(18-20 \mathrm{M} \Omega \cdot \mathrm{cm}^{-1}\right.$ at $\left.27^{\circ} \mathrm{C}\right)$ was used.

\section{Characterization}

SEM was carried out using a LEO 1430VP microscope. HRTEM and TEM were carried out using a FEI Tecnai G2 F20 transmission electron microscope, TF20 (200 kV). The X-ray powder diffraction patterns

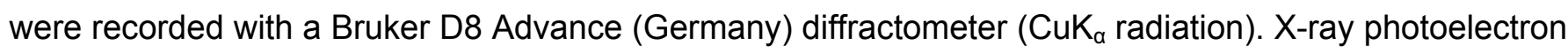
spectroscopy (XPS) measurements were performed by an X-Ray BesTec XPS system (Germany) with an AlK $_{\alpha} \mathrm{X}$-ray source (hu $=1,486.6 \mathrm{eV}$ ). Electrochemical experiments were performed using an EmStat3+ from PalmSens (Netherlands). The temperature during the measurements was measured by Laserliner 082 (Germany). FTIR spectra of the materials prepared as $\mathrm{KBr}$ pellets were recorded on a Bruker vector 22 in the range between 400 and $4000 \mathrm{~cm}^{-1}$. XAS measurements (EXAFS, XANES) were performed at the KMC-3 beamline at the BESSY II synchrotron facility (Helmholtz-Zentrum Berlin, Germany) at $20 \mathrm{~K}$ in a liquid-helium cooled cryostat (Oxford-Danfysik).

\section{Synthesis of MOF1}

Ultrathin NiCo-MOF (MOF 1) nanosheet was prepared according to the reported method by Zhao, et.al. ${ }^{[1]}$ First, DMF $(32 \mathrm{ml})$, ethanol $(2 \mathrm{ml})$ and water $(2 \mathrm{ml})$ were mixed in a $100 \mathrm{ml}$ tube. Next, $0.75 \mathrm{mmol}$ BDC was dissolved into the mixed solution under ultrasonication. Subsequently, 0.375 mmol $\mathrm{CoCl}_{2} \cdot 6 \mathrm{H}_{2} \mathrm{O}$ and $0.375 \mathrm{mmol} \mathrm{NiCl}_{2} \cdot 6 \mathrm{H}_{2} \mathrm{O}$ were added. After $\mathrm{Co}^{2+}$ and $\mathrm{Ni}^{2+}$ salts were dissolved, $0.8 \mathrm{~mL}$ TEA was quickly injected into the solution. Then, the solution was stirred for 5 min to obtain a uniform colloidal suspension. Afterwards, the colloidal solution was continuously ultrasonicated for $4 \mathrm{~h}(40 \mathrm{kHz})$ under air tight conditions. Finally, the products were obtained via centrifugation, washed with ethanol (3-5 times), and dried at room temperature. 


\section{Electrochemical measurements}

For the investigation of the electrochemical behavior of MOF 1, cyclic voltammetry (CV) in a threeelectrode cell was performed in which $\mathrm{Ag} / \mathrm{AgCl}(3.5 \mathrm{M} \mathrm{KCl})$, platinum sheet and FTO were used as the reference, the counter and the working electrode, respectively. $3.0 \mathrm{mg}$ of MOF 1 was added to $100 \mu \mathrm{L}$ water and dispersed by sonication and then this dispersed mixture was dropped on an FTO electrode (1.0 $\mathrm{cm} 2$ ) and dried in the ambient temperature. Then, $10 \mu \mathrm{L}$ of Nafion solution (10\% in methanol) was dropped on the catalyst surface and dried at room temperature. Cyclic voltammetry was performed in the range of

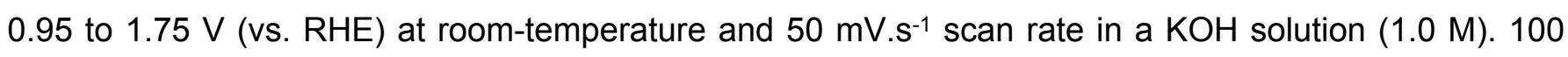
cycles of measurement were continued to follow the cluster behavior in long time operation. Chronoamperomtery was performed for MOF 1 sample by applying $1.65 \mathrm{~V}$ for one hour. After chronoamperomtrey, the solid phase was mechanically separated from the FTO surface and collected to analyze by different methods such as XAS, SEM, XRD, and FTIR.

\section{Oxygen-evolution measurements}

In-situ oxygen evolution activity of MOF 1 sample was measured using the same setup as aforementioned electrochemical system. The measurements were carried out at $25.0^{\circ} \mathrm{C}$ using an optical-probe oxygen meter (HQ40d from Hach, Düsseldorf, Germany). Electrolyte solution was degassed by purging with $\mathrm{Ar}$ gas. Then, the amount of oxygen was measured for $5 \mathrm{~min}$ before applying potential to be sure that there is no oxygen diffusion to the system. After making sure that there is no oxygen diffusion, chromnoamperometry was started at $1.65 \mathrm{~V}$ vs RHE and evolved oxygen measured simultaneously.

\section{XAS measurements}

$3.0 \mathrm{mg}(1.25 \mu \mathrm{mol})$ of MOF 1 was added to $100 \mu \mathrm{L}$ water and dispersed by sonication and then this dispersed mixture $(30 \mu \mathrm{L})$ was dropped on an FTO electrode $\left(1.0 \mathrm{~cm}^{2}\right)$ and dried in the ambient temperature. Then, $10 \mu \mathrm{L}$ of Nafion solution (10\% in methanol) was dropped on the catalyst surface and dried at room temperature. The amperometry measurement at $1.65 \mathrm{~V}$ vs RHE for one hour was performed in a solution of $\mathrm{KOH}(1.0 \mathrm{M})$ and by using $\mathrm{Ag} / \mathrm{AgCl}$ and platinum sheet as the reference and auxiliary electrodes, respectively. After amperometry, the sample was separated from the surface of FTO mechanically and prepared to be analyzed by XAS.

XAS measurements (EXAFS, XANES) at the nickel and cobalt K-edges for as-synthesized powder and after amperometry tests for 1.5 hours were performed at the KMC-3 beamline at the BESSY II synchrotron facility (Helmholtz-Zentrum Berlin, Germany). The measurements were performed at $20 \mathrm{~K}$ using a liquidhelium-cooled cryostat (Oxford-Danfysik) in the top-up mode of the BESSY II storage ring at $250 \mathrm{~mA}$ ring 
current. The angle between the sample surface and the incoming x-ray beam was $\sim 45^{\circ}$. The fluorescencedetected X-ray absorption spectra at the K-edge were collected using a 13-element Ge detector (UltraLEGe detector, Canberra $\mathrm{GmbH}$ ) installed perpendicular to the incident X-ray beam. 


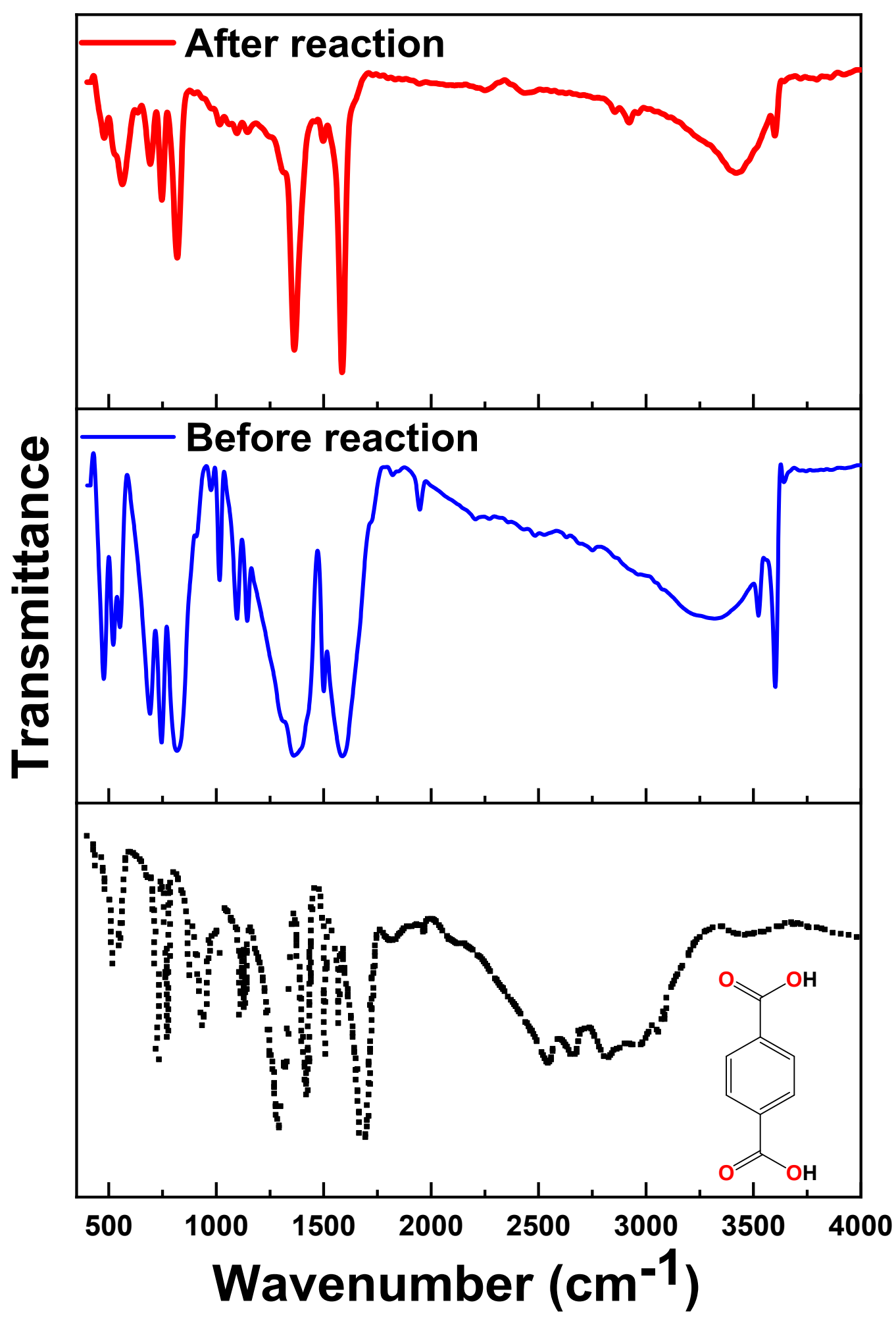

Figure S1. FTIR spectra of benzenedicarboxylic acid (black), MOF 1 (blue) and MOF 1 after one-hour amperometry at $1.65 \mathrm{~V}$ (red). 

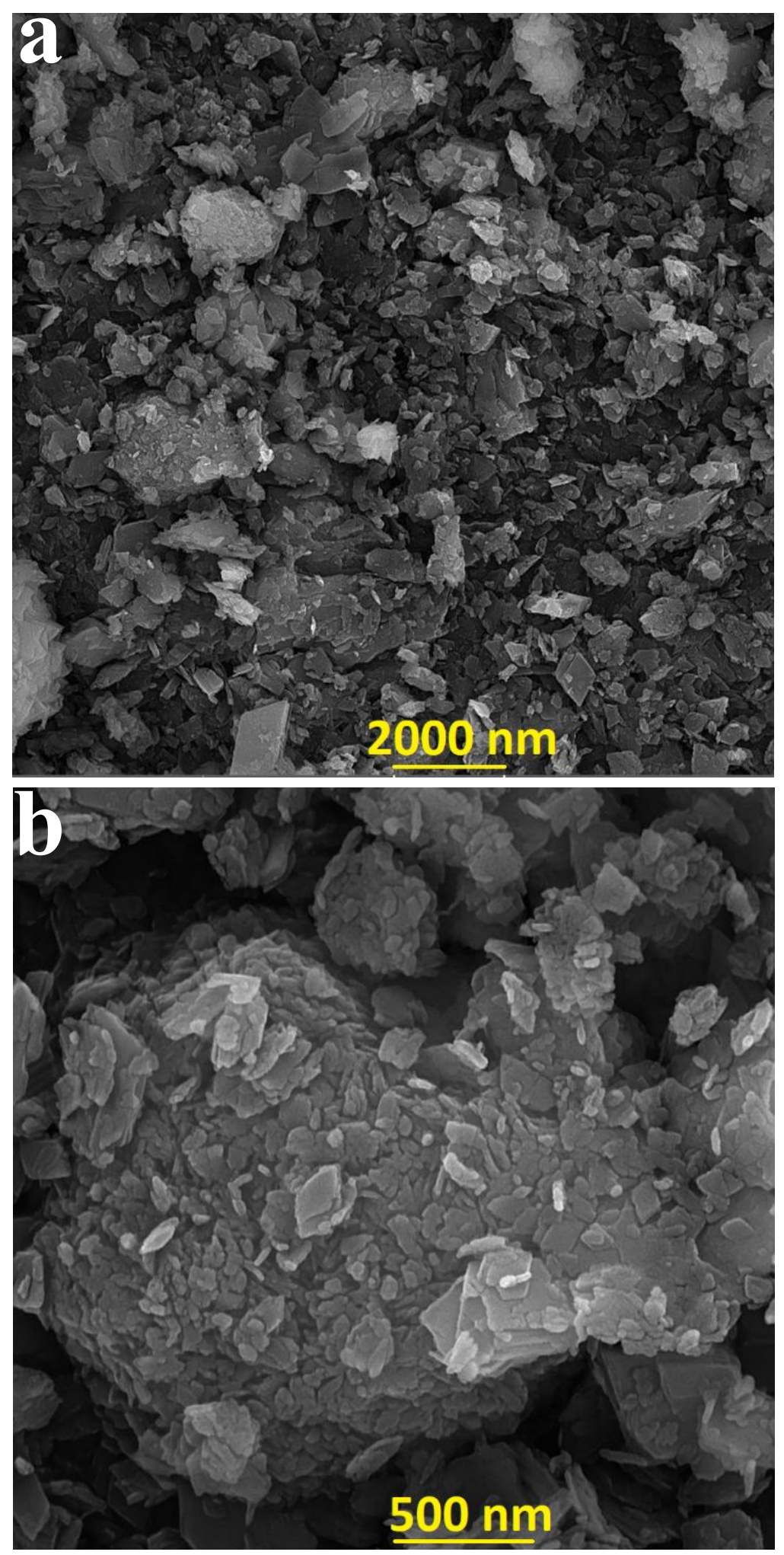

Figure S2. SEM images of MOF $\mathbf{1}$ at different magnifications $(\mathbf{a}, \mathbf{b})$. 

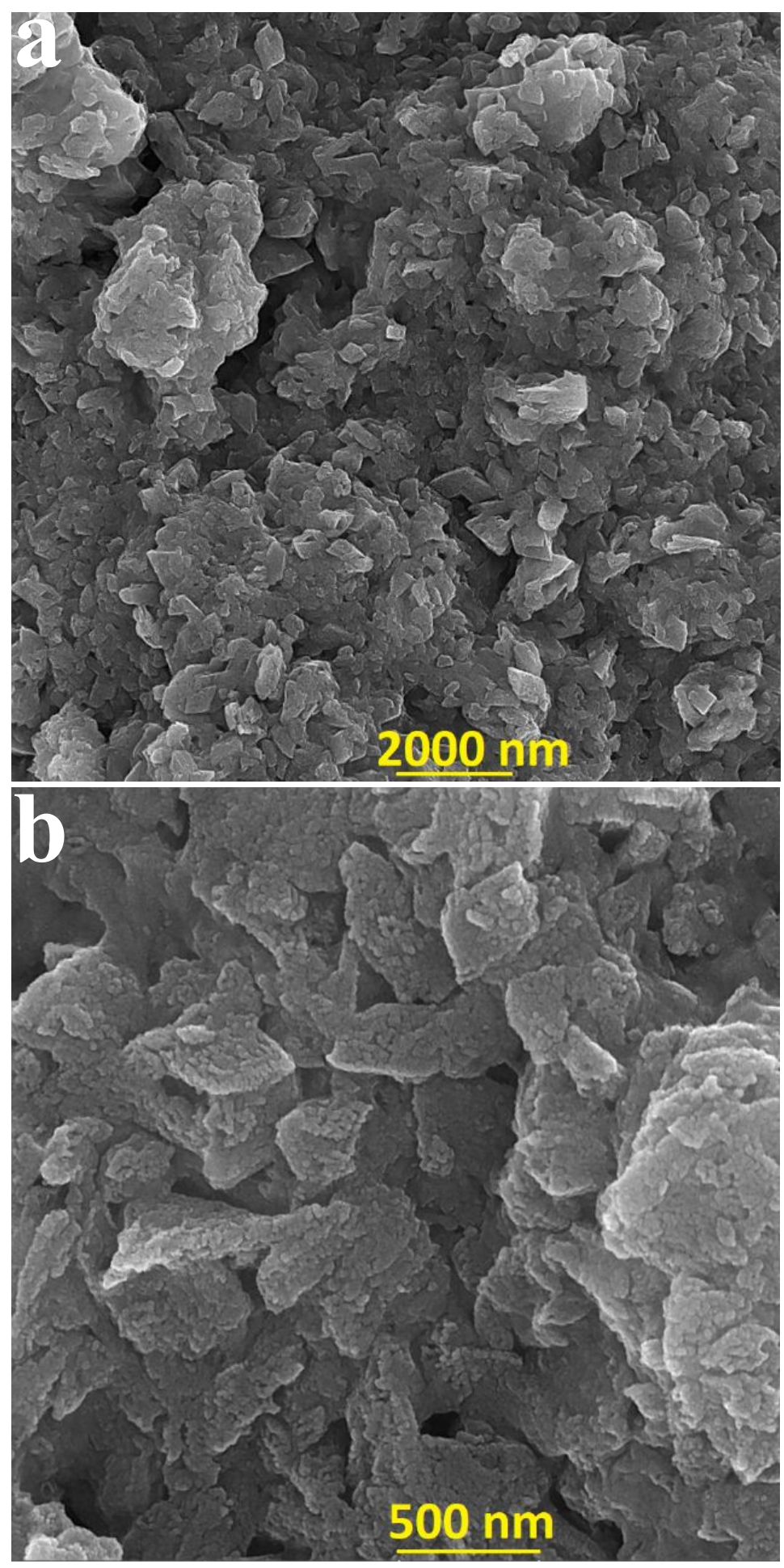

Figure S3. SEM images MOF 1 after one-hour amperometry at $1.65 \mathrm{~V}$ at different magnifications $(\mathbf{a}, \mathbf{b})$. 

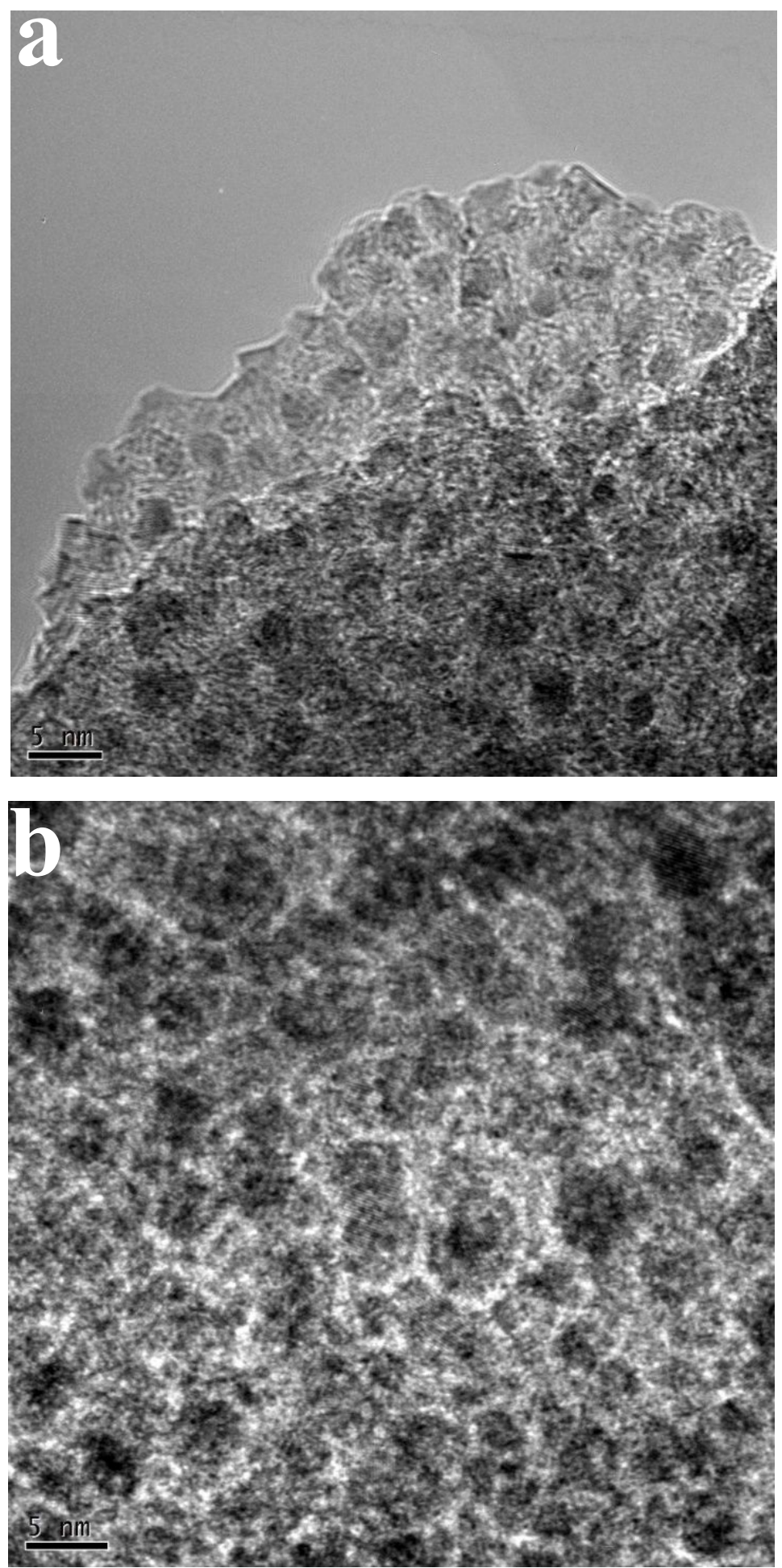

Figure S4. HRTEM images of MOF $\mathbf{1}$ at different magnifications $(\mathbf{a}, \mathbf{b})$. 

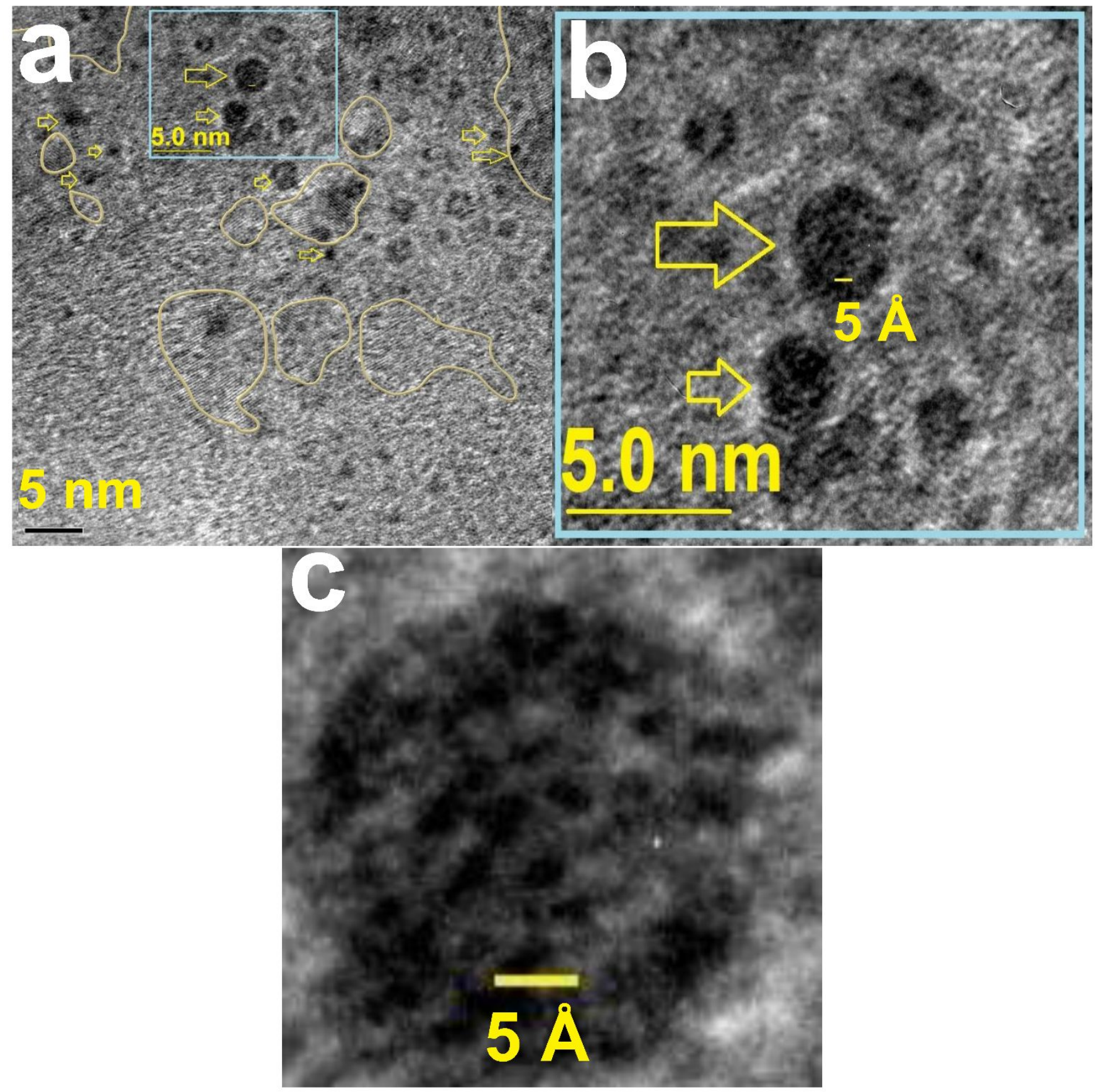

Figure S5. HRTEM images MOF 1 after one-hour amperometry at $1.65 \mathrm{~V}$ at different magnifications (a-c). Yellow areas show crystalline sections. 
a
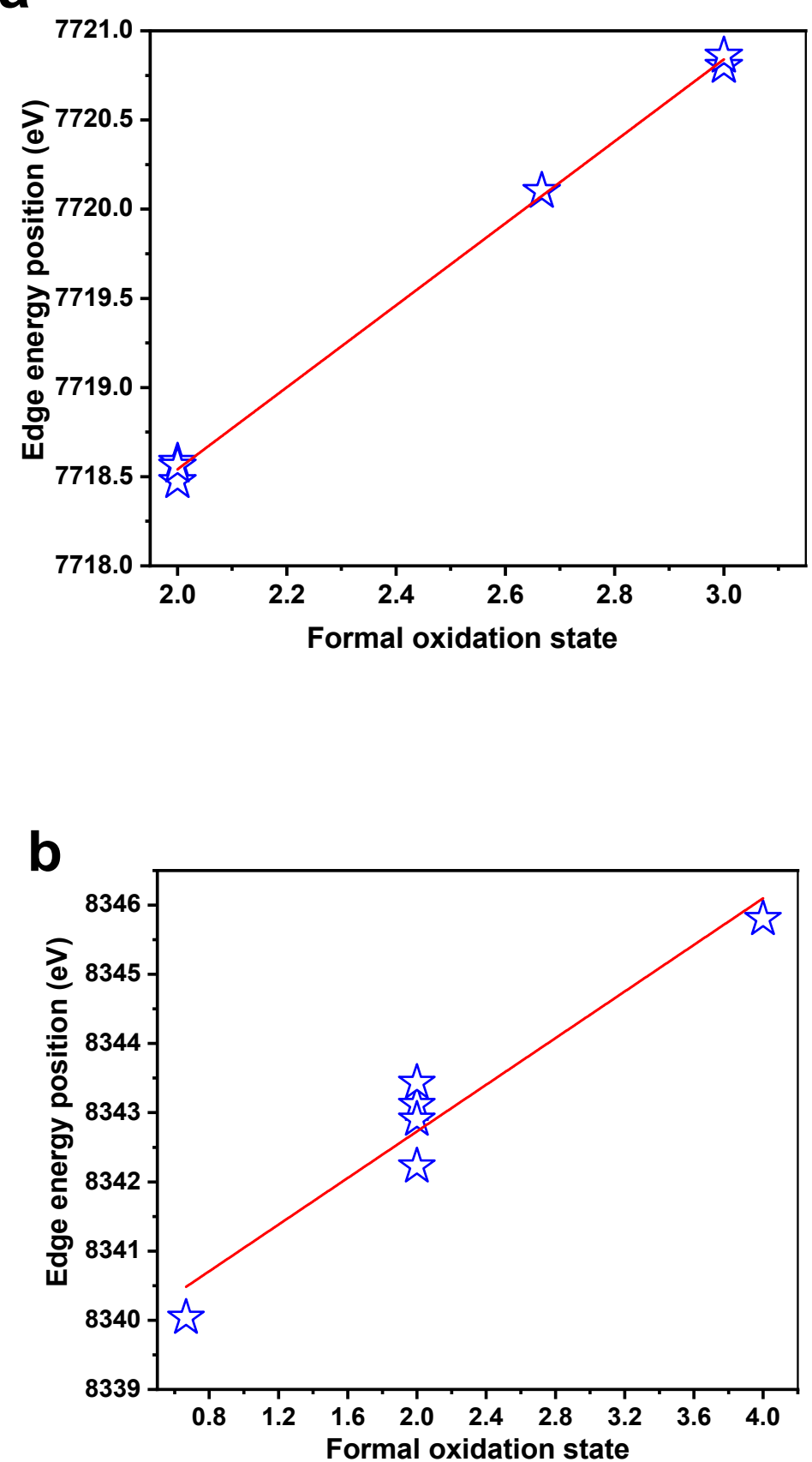

Figure S6. Co K-edge XANES of [Co" $\left.\left(\mathrm{H}_{2} \mathrm{O}\right)_{6}\right]\left(\mathrm{NO}_{3}\right)_{2}$ solution, [Co" $\left.\left(\mathrm{H}_{2} \mathrm{O}\right)_{6}\right]\left(\mathrm{NO}_{3}\right)_{2}$ powder, $\mathrm{Co}_{3}{ }_{3}\left(\mathrm{PO}_{4}\right)_{2}$ powder, Coll, III $_{3} \mathrm{O}_{4}$ powder, $\mathrm{Co}$ 'II' $\mathrm{OOH}$ powder, $\mathrm{LiCo}^{\prime \prime \prime} \mathrm{O}_{2}$ powder were measured as a reference and $\mathrm{Co}$ of the initial and operated samples (a). Ni $K$-edge XANES of $\mathrm{Ni}_{3} \mathrm{~S}_{2}, \mathrm{Nil}(\mathrm{OH})_{2}, \mathrm{Nil} \mathrm{O},\left[\mathrm{Ni}^{\prime \prime}\left(\mathrm{H}_{2} \mathrm{O}\right)_{6}\right]\left(\mathrm{NO}_{3}\right)_{2}, \mathrm{KNi}^{\prime V} \mathrm{IO}_{6}$ were measured as a reference and $\mathrm{Ni}$ of the initial and operated samples (b). 

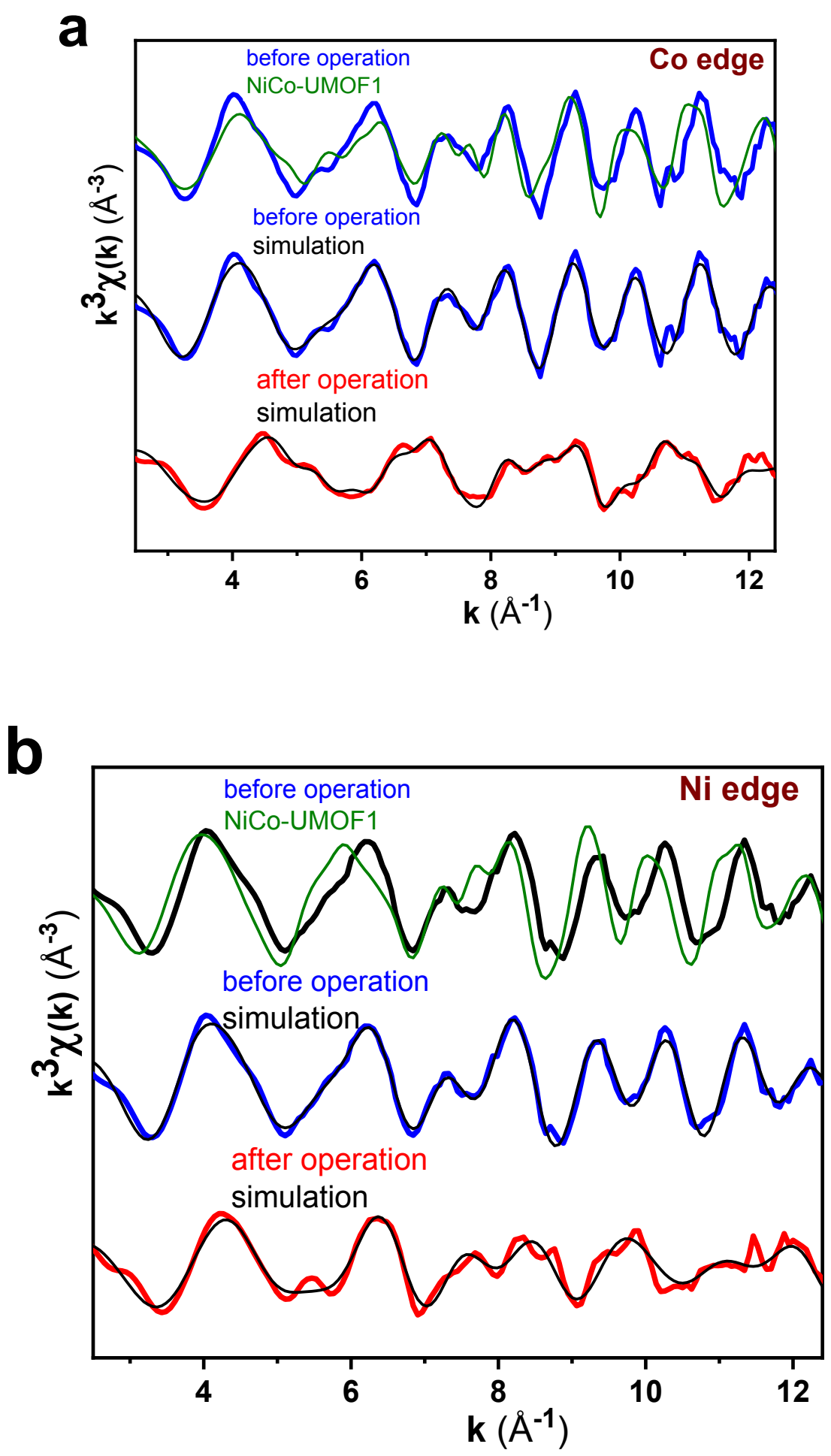

Figure S7. $\mathrm{k}^{3}$-weighted EXAFS oscillations of the NiCo-UMOF1 (ref. 1), MOF1 and MOF1 after operation. Thick black and blue lines show the experimental data, the thin red lines represent simulations. The fit parameters for the simulations are given in Table 1.

\section{Reference}


[1] S. Zhao, Y. Wang, J. Dong, C.-T. He, H. Yin, P. An, K. Zhao, X. Zhang, C. Gao, L. Zhang, Nat. Energy 2016, 1, 1-10. 